\title{
Oxidized unsaturated fatty acids induce apoptotic cell death in cultured cells
}

\author{
KATSUYA IUCHI $^{1}$, MIKA EMA ${ }^{1}$, MOE SUZUKI ${ }^{1}$, CHIKAKO YOKOYAMA $^{2}$ and HISASHI HISATOMI ${ }^{1}$ \\ ${ }^{1}$ Department of Materials and Life Science, Faculty of Science and Technology, Seikei University, Tokyo 180-8633; \\ ${ }^{2}$ Department of Biochemical Engineering, Graduate School of Science and Engineering, Yamagata University, \\ Yonezawa, Yamagata 992-8510, Japan
}

Received August 22, 2018; Accepted January 28, 2019

DOI: $10.3892 / \mathrm{mmr} .2019 .9940$

\begin{abstract}
Polyunsaturated fatty acids are oxidized by non-enzymatic or enzymatic reactions. The oxidized products are multifunctional. In this study, we investigated how oxidized fatty acids inhibit cell proliferation in cultured cells. We used polyunsaturated and saturated fatty acids, docosahexaenoic acid (DHA; 22:6), eicosapentaenoic acid (EPA; 20:5), linoleic acid (LA; 18:2), and palmitic acid (16:0). Oxidized fatty acids were produced by autoxidation of fatty acids for 2 days in the presence of a gas mixture $\left(20 \% \mathrm{O}_{2}\right.$ and $80 \% \mathrm{~N}_{2}$ ). We found that oxidized polyunsaturated fatty acids (OxDHA, OxEPA and OxLA) inhibited cell proliferation much more effectively compared with un-oxidized fatty acids (DHA, EPA and LA, respectively) in THP-1 (a human monocytic leukemia cell line) and DLD-1 (a human colorectal cancer cell line) cells. In particular, OxDHA markedly inhibited cell proliferation. DHA has the largest number of double bonds and is most susceptible to oxidation among the fatty acids. OxDHA has the largest number of highly active oxidized products. Therefore, the oxidative levels of fatty acids are associated with the anti-proliferative activity. Moreover, caspase-3/7 was activated in the cells treated with OxDHA, but not in those treated with DHA. A pan-caspase inhibitor (zVAD-fmk) reduced the cell death induced by OxDHA. These results indicated that oxidized products from polyunsaturated fatty acids induced apoptosis in cultured cells. Collectively, the switch between cell survival and cell death may be regulated by the activity and/or number of oxidized products from polyunsaturated fatty acids.
\end{abstract}

Correspondence to: Dr Katsuya Iuchi, Department of Materials and Life Science, Faculty of Science and Technology, Seikei University, 3-3-1 Kichijojikitamachi, Tokyo 180-8633, Japan E-mail: iuchi@st.seikei.ac.jp

Key words: polyunsaturated fatty acids, non-enzymatic oxidation, cell death, apoptosis, lipotoxicity

\section{Introduction}

Fatty acids are structural components of the cellular membrane, and a source of energy. Numerous studies have been conducted on fatty acids to analyze their effect on diseases and health $(1,2)$. Consumption of a high amount of unsaturated fatty acids is thought to be one of the main risk factors for certain cancers. Fatty acids, such as docosahexaenoic acid (DHA), eicosapentaenoic acid (EPA), linoleic acid (LA), and palmitic acid (PA), have various cellular roles. Especially, polyunsaturated fatty acids play a key role in inflammatory processes (3). In addition, some fatty acids suppress cancer growth in vitro and in vivo (4-10). A mixture of fatty acids (EPA+arachidonic acid (AA) or $\mathrm{DHA}+\mathrm{AA})$ decreases the viability and proliferation of breast cancer cell lines (MDA-MB-231 and MCF7) (11). Saturated fatty acids (PA and stearic acid) also induce death in human cancer cells $(12,13)$. Not only fatty acids, but also fatty acid-analogues have been shown to be potent in anti-cancer therapies (14). However, the mechanism of the multifunctional effects of fatty acids is not clear.

Polyunsaturated fatty acids are oxidized by non-enzymatic or enzymatic reactions. In non-enzymatic reaction, lipid peroxidation is an autoxidation process initiated by the attack of free radicals, such as reactive oxygen and nitrogen species $\left(\cdot \mathrm{OH}\right.$ and $\left.\mathrm{ONOO}^{-}\right)$. After a radical chain reaction, various bioactive oxidized products are produced from fatty acids (15). Paradoxically, these products exhibit both pro- and anti-inflammatory effects. The oxidized 1-palmitoyl-2-arachidonoyl-sn-glycero-3-phosphocholine (OxPAPC) physiologically exerts the multiple functions $(15,16)$.

15 -Deoxy- $\Delta^{12,14}$-prostaglandin $\mathrm{J}_{2}$, an oxidized fatty acid, induces death in breast cancer cells (17). Synthetic ether phospholipid hexadecyl azelaoyl phosphatidylcholine causes mitochondrial dysfunction and apoptosis in HL-60 cells (18). 13-Hydroxy ocatadecadienoic acid is a highly potent inducer of reactive oxygen species (ROS) production in human aortic endothelial cells (19). DHA hydroperoxide is a potential inducer of apoptosis via mitochondrial dysfunction in human neuroblastoma SH-SY5Y cells (20). In contrast, 15 -deoxy- $\Delta^{12,14}$-prostaglandin $\mathrm{J}_{2}$ protects $\mathrm{SIN}$-1-induced death in PC12 cells (21). EPA, but not AA, attenuates dexamethasone-induced cell apoptosis in murine bone marrow-derived mesenchymal stem cells (22). The mixture of OxEPA 
and OxDHA ameliorates the loss of viability induced by 2,2', 4,4'-tetrabromodiphenyl ether in HepG2 cells (23). Oleic acid (0.1-1.5 mM) has a protective effect against the cytotoxicity induced by tert-butyl hydroperoxide, a oxidative stress inducer, in mouse 3T3-L1 fibroblasts, while DHA (>0.1 mM) enhanced this cytotoxicity (24). However, the relationship between oxidized fatty acids and cell death is controversial.

In this study, we aimed to investigate how oxidized fatty acids affect the viability and death of cultured cells. We showed that of all the fatty acids used in this study, OxDHA had the most anti-proliferative effect in cultured cells. Moreover, OxDHA, but not DHA induced apoptosis through caspase activation in THP-1 cells (human monocytic leukemia cell line). Among fatty acids, DHA has the most number of double bonds, and is most susceptible to oxidation. These results suggested that oxidized products from highly unsaturated fatty acids have a potent anti-proliferative and pro-apoptotic activity. The switch between cell survival and cell death may be regulated by the activity and/or number of oxidized products from polyunsaturated fatty acids.

\section{Materials and methods}

Cell culture. THP-1 (ATCC) and DLD-1 (Cell Resource Center) cells were cultured in RPMI medium (Invitrogen/Life technologies, Tokyo, Japan) supplemented with $10 \%$ fetal bovine serum (GE Healthcare Life Sciences, Tokyo, Japan), penicillin (Nacalai Tesque, Kyoto, Japan), streptomycin (Nacalai Tesque, Kyoto, Japan), and amphotericin B (GE Healthcare Life Sciences, Tokyo, Japan) at $37^{\circ} \mathrm{C}$ with $5 \% \mathrm{CO}_{2}$.

Preparation of human lymphocytes. Cell culture analysis, using peripheral blood mononuclear cells (PBMC) from healthy volunteers, was approved by the Institutional Review of Committee of Seikei University. Informed consent was obtained from volunteers in accordance with the Declaration of Helsinki. Peripheral blood samples from four healthy volunteers were transferred into polystyrene centrifuge tubes containing EDTA and then gently mixed. The same volume of PBS was added into the tubes. The diluted blood samples ( $3 \mathrm{ml}$ each) were layered on $3 \mathrm{ml}$ of Lymphosepar I (Immuno-Biological Laboratories, Gunma, Japan). Lymphocytes were isolated by centrifugation at room temperature for $30 \mathrm{~min}$ at $1,800 \mathrm{rpm}(400 \mathrm{x} \mathrm{g})$. The lymphocyte layer was collected and washed with PBS, and then resuspended in RPMI-1640 medium supplemented with $10 \%$ fetal bovine serum, penicillin, streptomycin, and amphotericin B. Lymphocytic blastogenesis was induced by treatment with $20 \mu \mathrm{g} / \mathrm{ml}$ phytohemagglutinin-P (PHA) (Sigma-Aldrich, Tokyo, Japan). PHA is able to stimulate lymphocyte proliferation by binding on the cell surface and activating RNA and protein synthesis.

Oxidized fatty acid preparation in an in vitro cell free system. Oxidized fatty acids were produced by autoxidation as described previously (25). DHA, EPA, LA, and PA were purchased from Funakoshi (Tokyo, Japan). $2.5 \mathrm{mg}$ of DHA, EPA, LA, or PA in $500 \mu 1$ of ethanol was transferred to a glass tube, and dried under a gentle stream of nitrogen. The fatty acid residue was allowed to autoxidize for 2 days in the presence of the gaseous mixture of $80 \% \mathrm{~N}_{2}$ and $20 \% \mathrm{O}_{2}$ at $25^{\circ} \mathrm{C}$ in a closed aluminum bag (Fig. 1). Then, the oxidized fatty acids were suspended in PBS containing $10 \%$ ethanol.
Treatment of cells with fatty acids or oxidized fatty acids. Cells were seeded at a concentration of $0.5-1 \times 10^{5}$ cells $/ \mathrm{ml}$, incubated for the indicated times, and then treated with the fatty acids or oxidized fatty acids at the indicated concentrations. We used the concentrations of fatty acids used in our experiments ( 0.6 to $5 \mu \mathrm{g} / \mathrm{ml}$ ), based on the previous reference (26) described the human plasma concentrations of DHA.

Cellular viability of cells treated with oxidized fatty acids. Cell viability was measured using a Cell Counting Kit-8 (CCK-8, Dojindo Laboratories, Kumamoto, Japan), according to the manufacture's protocol. The cells were treated with oxidized fatty acids. Then, the CCK- 8 reagent was added and the cells were incubated for $4 \mathrm{~h}$ at $37^{\circ} \mathrm{C}$. Absorbance was measured at $450 \mathrm{~nm}$ using a microplate reader (Food Mark, Bio-Lad, CA, USA).

Caspase-3/7 activation assay. To determine the activity of caspase-3/7, CellEvent ${ }^{\mathrm{TM}}$ Caspase-3/7 Green ReadyProbes reagent (Invitrogen/Life Technologies, Tokyo, Japan) was used. This reagent consists of a four-amino acid peptide (DEVD) conjugated to a nucleic acid-binding dye. After $1 \mathrm{~h}$ of incubation, the fluorescence of the nuclei was observed using a fluorescence microscope (Leica DM IL LED; Leica Microsystems, Wetzlar, Germany).

Propidium iodide exclusion assay. Cell death was determined by the ability of the cells to exclude propidium iodide (PI) (Sigma-Aldrich). THP-1 cells were treated with vehicle, DHA, or OxDHA in the absence or presence of $20 \mu \mathrm{M} z V A D-f m k$ (American Peptide Company, CA, USA), and stained with $50 \mu \mathrm{g} / \mathrm{ml}$ PI. The cells were then analyzed using the Cell Lab Quanta $^{\mathrm{TM}}$ SC flow cytometer (Beckman Coulter, Brea, CA, USA).

Determination of apoptotic cells treated with OxDHA. Apoptotic cell death assay was performed by Hoechst 33342 and propidium iodide staining, as described previously (27). The cells were treated with oxidized fatty acids for the indicated times. The cells were stained with Hoechst 33342 (1 $\mu \mathrm{g} / \mathrm{ml}$ ) (Dojindo Laboratories, Kumamoto, Japan) and propidium iodide $(1 \mu \mathrm{g} / \mathrm{ml})$, and observed under a fluorescence microscope (Leica DM IL LED; Leica Microsystems). The apoptotic cell death was determined by morphological changes of chromatin (condensed and/or fragmented chromatin).

Assessment of apoptotic cell death by Annexin V staining. After treatment with OxDHA, THP-1 cells were washed with PBS and resuspended in a staining solution containing the Annexin V-Cy3 reagent (BioVision, CA, USA) in 1xbinding buffer. After a 15-min incubation in the dark at room temperature, the cells were washed with HEPES buffer, and observed using a fluorescence microscope (Leica DM IL LED; Leica Microsystems).

Statistical analysis. The data shown in the figures represent the mean values \pm standard deviation of three or four independent determinations. Data were evaluated statistically by one-way analysis of variance followed by the Tukey's honest significant difference test. The statistical significance at different P-values is indicated in each figure. 
<smiles>CC/C=C\C/C=C\C/C=C\C/C=C\C/C=C\C/C=C\CCC(=O)O</smiles><smiles>CC/C=C\C/C=C\C/C=C\C/C=C\C/C=C\CCCC(=O)O</smiles>

Eicosapentaenoic acid (EPA; 20:5)<smiles>CCCCC/C=C\C/C=C\CCCCCCCC(=O)O</smiles><smiles>CCCCCCCCCCCCCCCC(=O)O</smiles>

B

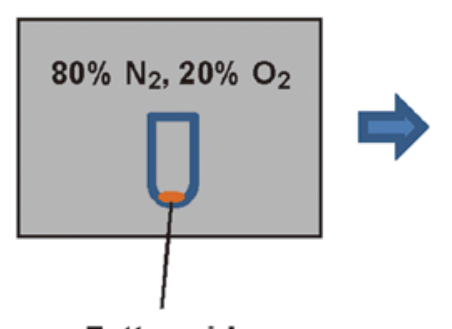

Fatty acids

(DHA, EPA, LA, PA)
OXDHA $25^{\circ} \mathrm{C}, 2$ days Autoxidation
OXEPA

OXLA OXPA

Figure 1. Structure of fatty acids and scheme for fatty acid oxidation. (A) Structure of polyunsaturated and saturated fatty acids. Scheme for air oxidation of fatty acid. (B) Fatty acids were autoxidized for 2 days in the presence of $80 \% \mathrm{~N}_{2}$ and $20 \% \mathrm{O}_{2}$. Ox, oxidized.

\section{Results}

Oxidized polyunsaturated fatty acids inhibit proliferation of cultured cells. Previous studies showed that polyunsaturated fatty acids inhibit cancer cell growth in vitro (28). We first investigated the effect of fatty acids and oxidized fatty acids on the proliferation of various cultured cells, as determined by the CCK-8 assay (Figs. 2 and 3). Treatment with OxDHA significantly decreased the proliferation of THP-1 cells in a dose-dependent manner (Fig. 2A). Native DHA slightly decreased cell proliferation at high concentrations $(>2.5 \mu \mathrm{g} / \mathrm{ml}$ DHA). OxEPA also decreased the proliferation of THP-1 cells dose-dependently, but EPA (except for $5.0 \mu \mathrm{g} / \mathrm{ml}$ EPA) did not (Fig. 2B). OxLA, as well as OxEPA, slightly decreased the proliferation of THP-1 cells dose-dependently, but LA (except for $5.0 \mu \mathrm{g} / \mathrm{ml} \mathrm{LA}$ ) did not (Fig. 2C). Neither PA nor OxPA inhibited the proliferation of THP-1 cells (Fig. 2D). As shown in Fig. 3, OxDHA but not DHA inhibited the proliferation of the DLD-1 cells. Proliferation in DLD-1 cells was hardly inhibited by EPA, LA, OxEPA, and OxLA, even at high concentrations $(5.0 \mu \mathrm{g} / \mathrm{ml})$ (Figs. 3B and 3C). PA and OxPA hardly decreased the proliferation of DLD-1 cells at all concentrations (Fig. 3C). As shown in Figs. 2 and 3, OxDHA had the most anti-proliferative effect among these fatty acids. These results indicated that the anti-proliferative effect of oxidized fatty acids is responsible for the activity and/or number of oxidized products.

Oxidized DHA, but not DHA induces death of THP-1 cells. As shown above, treatment of cells with oxidized unsaturated fatty acids resulted in a decrease in their proliferation. To investigate whether the oxidized fatty acids induced death in the cultured cells, the THP-1 cells were analyzed using the propidium iodide (PI) exclusion assay. PI can only enter the dead cells, as their membranes are disrupted. As described in Fig. 4A, samples treated with OxDHA, but not with DHA contained PI-positive cells (dead cells). OxDHA-induced cell death was significantly suppressed by a pan-caspase inhibitor (zVAD-fmk) (Fig. 4A). As presented in Fig. 4B, the anti-proliferative effect of OxDHA was partially suppressed by zVAD-fmk (Fig. 4B). Palmitic acid did not inhibit cell proliferation in the presence of zVAD-fmk (Fig. 4C). In both the cell proliferation and the PI exclusion assay, the OxDHA-induced decrease of viability was suppressed by pre-treatment with zVAD-fmk. These results suggest that OxDHA decreased cell proliferation through the activation of caspases, followed by cell death.

Oxidized DHA, but not DHA induces apoptosis in THP-1 cells. During early apoptosis, phosphatidylserine on the outer membrane of cells was exposed. As shown in Fig. 5A, 
A
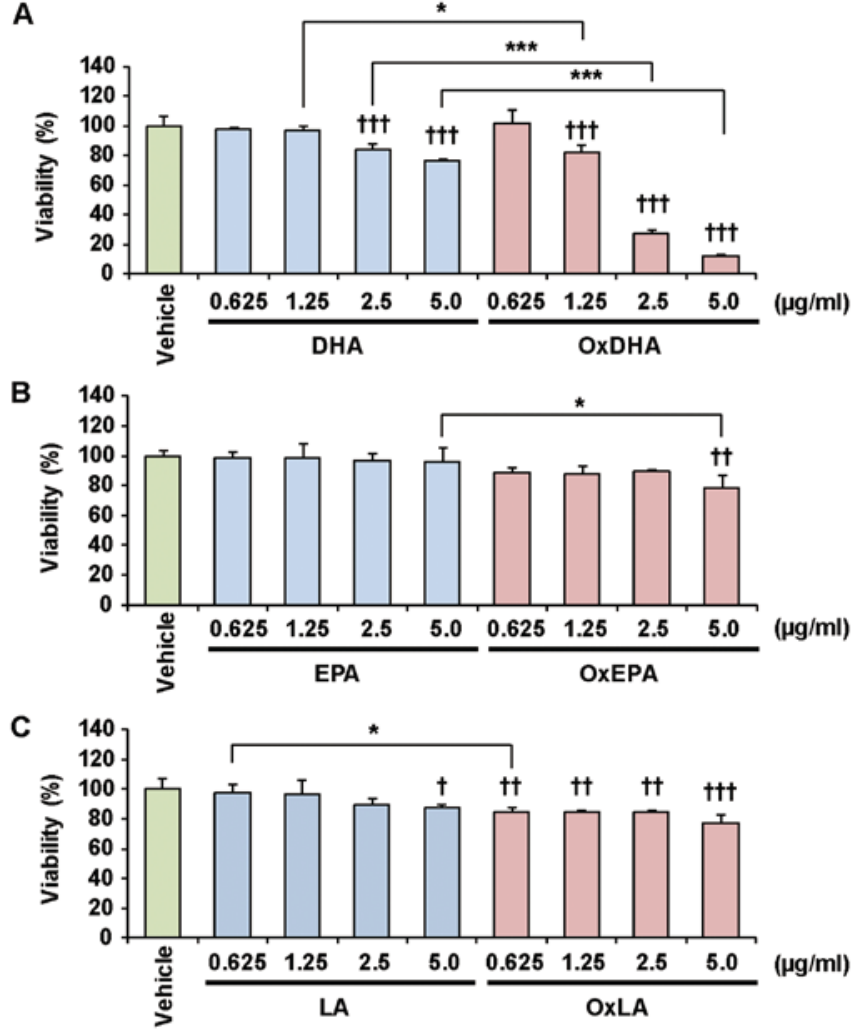

D

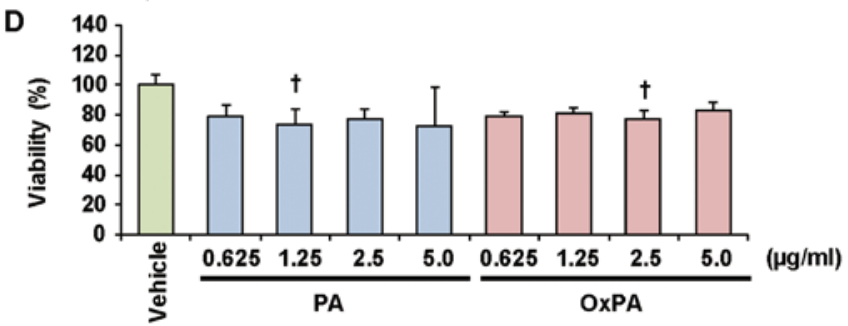

Figure 2. Effect of FA and OxFA on THP-1 cell proliferation. (A) Effect of DHA or OxDHA on cell proliferation. THP-1 cells were treated with DHA or OxDHA at the indicated concentrations for $24 \mathrm{~h}$. Cell growth was determined by a Cell Counting Kit- 8 assay, according to the manufacturer's protocol. (B) Effect of EPA or OxEPA on cell proliferation. (C) Effect of LA or OxLA on cell proliferation. (D) Effect of PA or OxPA on cell proliferation. $n=3-4$. ${ }^{\dagger} \mathrm{P}<0.05,{ }^{\dagger} \mathrm{P}<0.01,{ }^{\dagger \dagger} \mathrm{P}<0.001$ vs. vehicle; ${ }^{*} \mathrm{P}<0.05,{ }^{* * * *} \mathrm{P}<0.001$. FA, fatty acid; Ox, oxidized; DHA, docosahexaenoic acid; EPA, eicosapentaenoic; LA, linoleic acid; PA, palmitic acid.

the analysis using the Annexin V-Cy3 reagent, which stains phosphatidylserine residues, showed that the number of Annexin V-positive cells was high in the OxDHA-treated samples (Fig. 5A). Apoptosis is generally accompanied by chromatin shrinkage, chromatin condensation, and nuclear fragmentation. We analyzed the changes in chromatin morphology in THP-1 cells treated with OxDHA. As shown in Fig. 5B, THP-1 cells treated with OxDHA but not DHA or PA exhibited typical apoptotic features, such as chromatin condensation and fragmentation (Fig. 5B). These results suggested that OxDHA induced apoptotic cell death.

Oxidized DHA but not DHA induces caspase-3/7 activation in THP-1 cells. Fig. 4 showed that OxDHA-induced cell death was inhibited by a pan-caspase inhibitor. Next, we investigated whether the members of the caspase family are required for the induction of apoptosis by OxDHA. Caspase-3/7 were activated
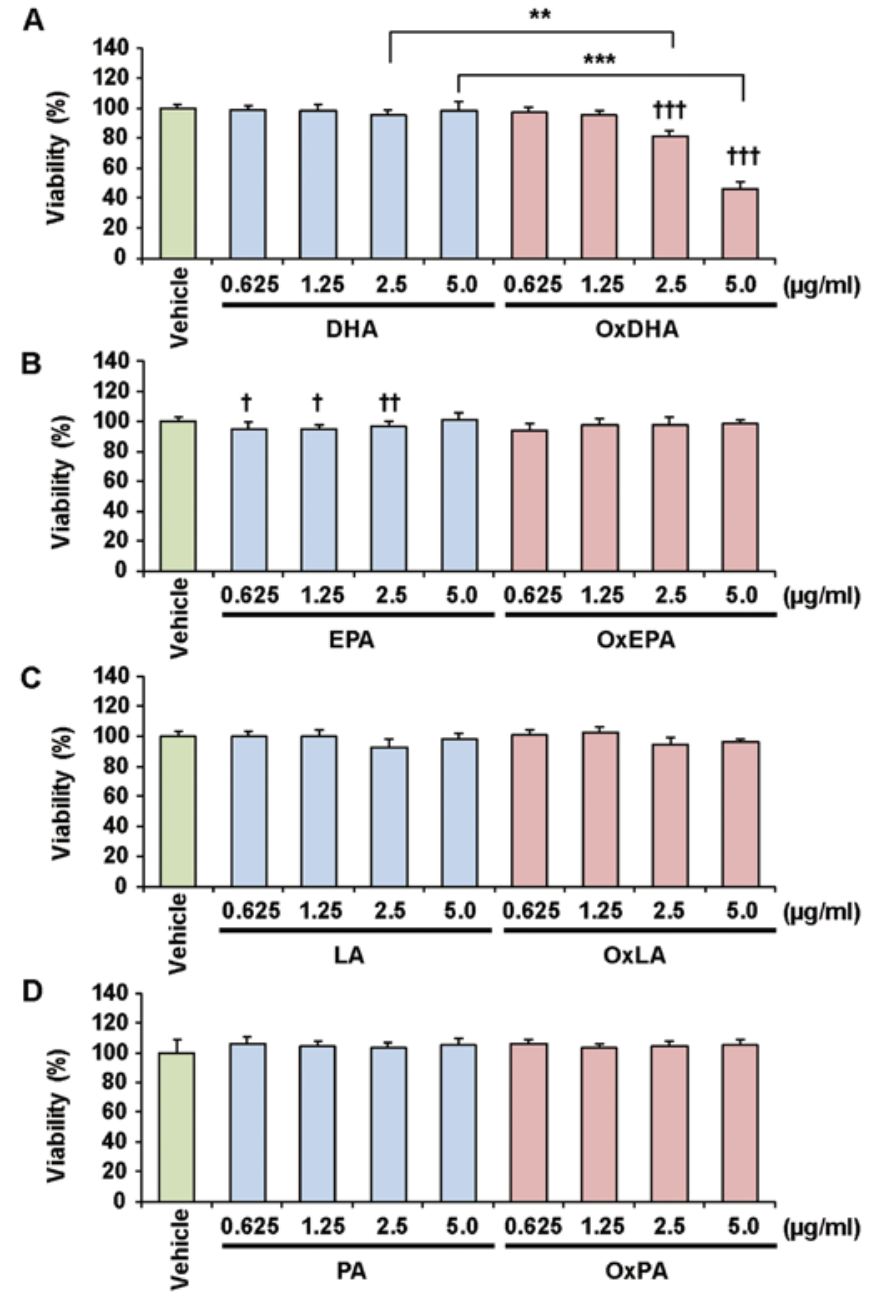

Figure 3. Effect of FA and OxFA on DLD-1 cell proliferation. (A) Effect of DHA or OxDHA on cell proliferation. DLD-1 cells were treated with DHA or OxDHA at the indicated concentrations for $24 \mathrm{~h}$. Cell growth was determined by a Cell Counting Kit-8 assay. (B) Effect of EPA or OxEPA on cell proliferation. (C) Effect of LA or OxLA on cell proliferation. (D) Effect of PA or OxPA on cell proliferation. $\mathrm{n}=4 .{ }^{\dagger} \mathrm{P}<0.05,{ }^{\dagger \dagger} \mathrm{P}<0.01,{ }^{\dagger \dagger} \mathrm{P}<0.001$ vs vehicle; ${ }^{* *} \mathrm{P}<0.01,{ }^{* * *} \mathrm{P}<0.001$. FA, fatty acid; Ox, oxidized; DHA, docosahexaenoic acid; EPA, eicosapentaenoic; LA, linoleic acid; PA, palmitic acid.

in THP-1 cells treated with OxDHA; this was not observed in THP-1 cells treated with DHA and vehicle (Fig. 6). These results indicate that caspase-3/7 activation is involved in apoptotic cell death induced by $\mathrm{OxDHA}$.

\section{Discussion}

In this study, the oxidized products from unsaturated fatty acids (DHA, EPA, and LA) exhibited an anti-proliferative activity in cultured human cells. Among the oxidized fatty acids, OxDHA had the most significant anti-proliferative activity. The numbers of double bonds of DHA, EPA, LA, or PA are 6, 5, 2, or 0, respectively (Fig. 1A). DHA has the most number of double bonds, and is most susceptible to oxidation. DHA has six double bonds and bis-allylic methylene groups, which have several possible positions for hydrogen abstraction. Therefore, DHA can be easily oxidized into highly active products in the air as well as under intracellular conditions. These chemical properties are the reason why OxDHA produces more toxic products 

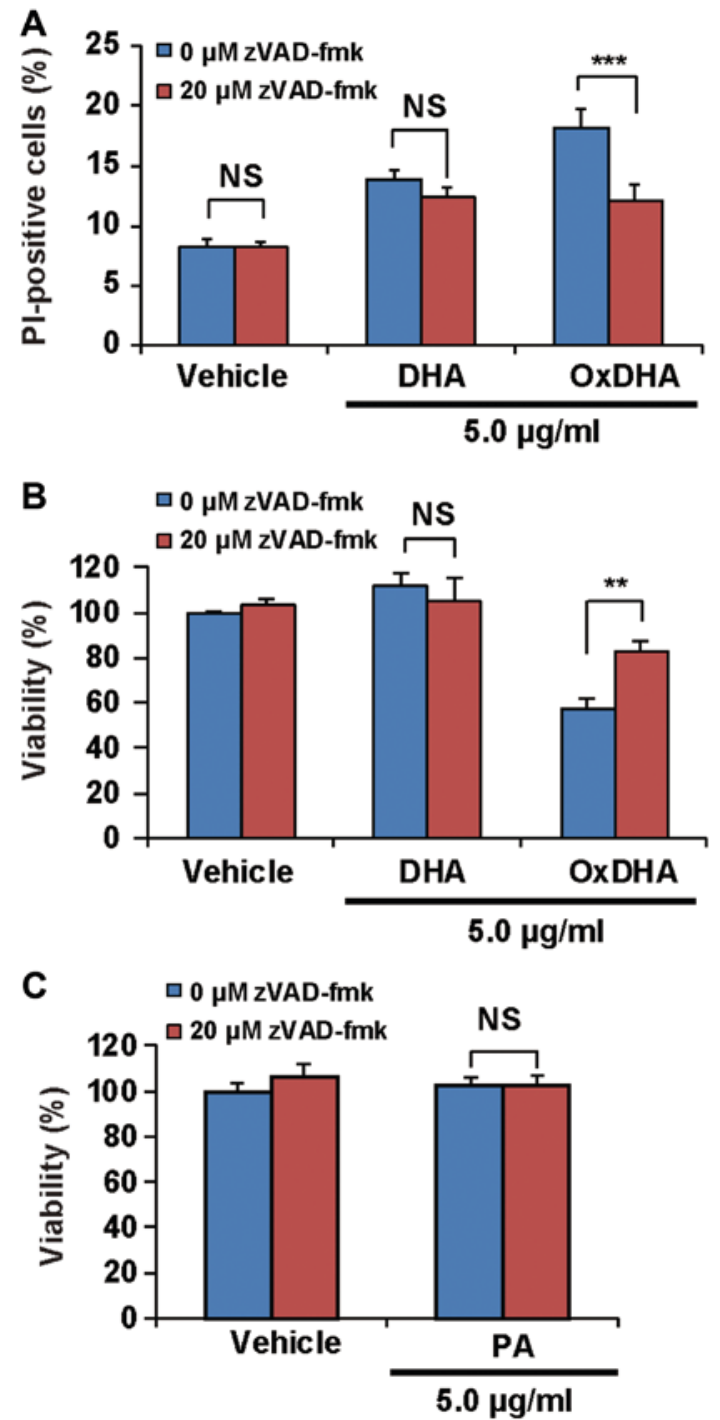

Figure 4. OxDHA induces cell death through caspase activation in THP-1 cells. (A) THP-1 cells were treated with vehicle, DHA, or OxDHA in the presence or absence of zVAD-fmk for $24 \mathrm{~h}$. Subsequently, cell death was assessed by PI staining. The THP-1 cells treated with vehicle, DHA, or OxDHA were analyzed for cell death using flow cytometry. (B) THP-1 cells were treated with vehicle, DHA, or OxDHA in the presence or absence of zVAD-fmk for $24 \mathrm{~h}$. Cell growth was determined by a CCK-8 assay. $n=3-4$. (C) THP-1 cells were treated with vehicle or PA in the presence or absence of zVAD-fmk for $24 \mathrm{~h}$. Cell growth was determined by CCK- 8 assay. $\mathrm{n}=4 .{ }^{* *} \mathrm{P}<0.01,{ }^{* * *} \mathrm{P}<0.001$. Ox, oxidized; DHA, docosahexaenoic acid; PI, propidium iodide; CCK-8, Cell Counting Kit-8; PA, palmitic acid.

(e.g., various hydroperoxides, aldehydes, and other products) than OxEPA and OxLA. Among these fatty acids, OxDHA has the most number of highly active oxidized products.

Suspension cells (THP-1 cells and lymphocytes) were sensitive to native and oxidized fatty acids (Figs. 2 and 7). However, only OxDHA induced a loss of viability of DLD-1 cells, which are adherent cells. Neither DHA nor OxDHA inhibited proliferation in Human Hepatoma HepG2 cells (data not shown). These results suggest that the sensitivity of blood cells to oxidized fatty acids is higher than those of solid cancer cells. In general, human leukemia cell lines are highly sensitive to ROS-generating agents.

As mentioned previously, OxDHA has the most potent anti-proliferative activity. DHA has the most number of double
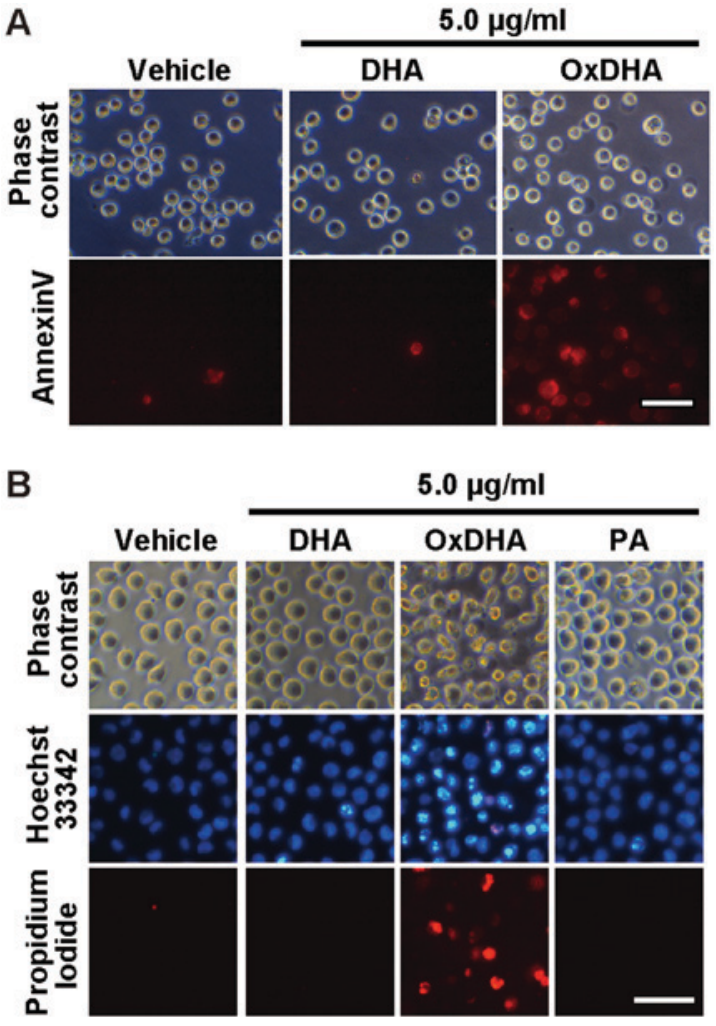

Figure 5. OxDHA induces apoptosis of THP-1 cells. (A) THP-1 cells were treated with vehicle, $\mathrm{DHA}$, or OxDHA for $24 \mathrm{~h}$ at the indicated concentrations. Subsequently, cell death was assessed using Annexin V assay. (B) Apoptotic nuclear morphological changes of THP-1 cells treated with OxDHA. THP-1 cells were treated with vehicle, DHA, OxDHA, or PA for $48 \mathrm{~h}$ at the indicated concentrations. Then, the cells were stained with Hoechst 33342 Propidium Iodide and observed under a fluorescence microscope. Apoptotic cells had condensed or fragmented chromatin. Scale bar, $50 \mu \mathrm{m}$. Ox, oxidized; DHA, docosahexaenoic acid; PA, palmitic acid.

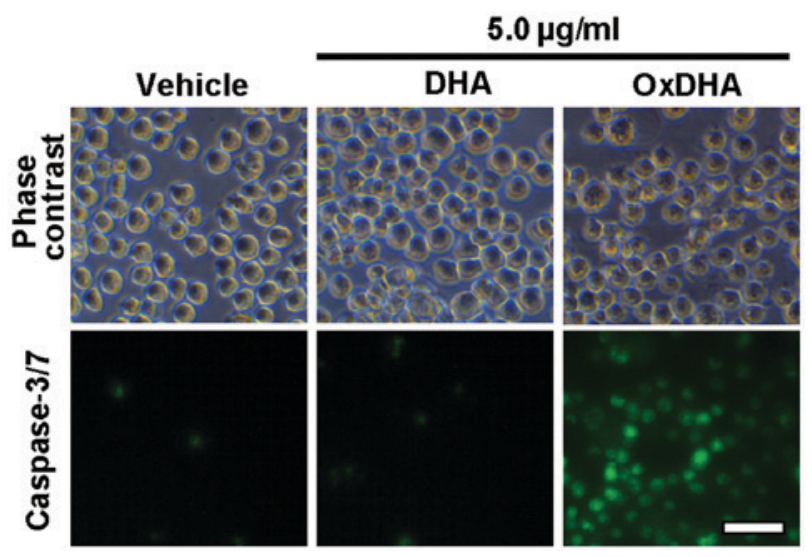

Figure 6. OxDHA induces caspase-3/7 activation in THP-1 cells. THP-1 cells were treated with vehicle, DHA, or OxDHA for $24 \mathrm{~h}$ at the indicated concentrations. Then, apoptotic cell death was detected by CellEvent caspase-3/7 Green Detection reagent, according to the manufacturer's protocol. Scale bar, $50 \mu \mathrm{m}$. Ox, oxidized; DHA, docosahexaenoic acid.

bonds; therefore, it has the most unsaturated fatty acids and sensitivity to ROS. In contrast, saturated fatty acids, such as PA, are resistant to oxidation when exposed to ROS. As shown in Figs. 2 and 3, neither PA nor OxPA had an anti-proliferative activity in this study. To date, the oxidation of unsaturated fatty acids generates many diverse oxidized products, such 


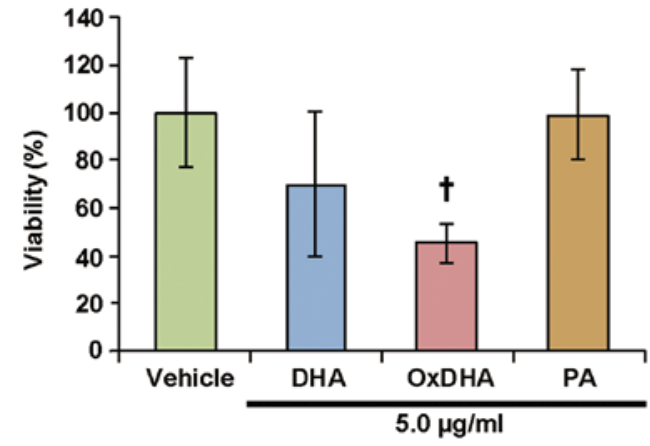

Figure 7. Effect of DHA and OxDHA on viabiltity of human lymphocytes. Human lymphocytes were treated with vehicle, DHA, OxDHA or PA for $24 \mathrm{~h}$ at the indicated concentrations. Cell growth was determined by Cell Counting Kit-8 assay, according to the manufacturer's protocol. $n=3-4$. ${ }^{\dagger} \mathrm{P}<0.05$ vs. vehicle. Ox, oxidized; DHA, docosahexaenoic acid; PA, palmitic acid.

as 4-hydroxy-2-nonenal (HNE), malondialdehyde (MDA), acrolein, 13-hydroperoxylinoleic acid (13-HPODE), and $\mathrm{F}_{2}$-isoprostanes (29-33). In the present study, OxDHA and OxEPA had a light-yellow color, but native DHA and EPA did not (data not shown). It is reported that DHA is oxidized by oxidizing agents such as 2,2'-azobis- (2-amidinopropane) hydrochloride (AAPH) or copper ions. In the chemical reaction, a family of $\mathrm{F}_{4}$-isoprostanes were produced from DHA (34). A4/J4-neuroprostanes were identified by the mass spectrometry analysis of AAPH-treated DHA, which has a cytoprotective effect and an anti-inflammatory activity (35). Previously, using mass spectroscopy, we had described that a large number of bioactive oxidized lipids were produced by the autoxidation of PAPC in an in vitro cell-free system (25). Therefore, the OxDHA used in this study may also contain various bioactive oxidized fatty acids. The anti-proliferative activity of native polyunsaturated fatty acids may be dependent on their oxidized products in cells.

$15 \mathrm{~d}_{-} \mathrm{PGJ}_{2}$, a potent inhibitor of mitochondrial respiratory complex I, increases the rate of ROS production (36). Therefore, OxDHA may inhibit mitochondrial respiratory complexes. DHA induced apoptotic cell death through ROS production and caspase- 8 activation (37). In our study, caspase-3/7 was activated in THP-1 cells treated with OxDHA. In general, the caspase-3/7 is activated by mitochondrial dysfunction followed by caspase- 8 and/or caspase- 9 activation. Collectively, these results and reports suggest that $\mathrm{OxDHA}$ may activate caspase- 8 and/or caspase-9 via mitochondrial dysfunction.

DHA blocks the NF- $\kappa$ B pathway and significantly decreases cell proliferation (38). 13-HPODE upregulates the expression of the chemokine monocyte chemoattractant protein-1 via the activation of $\mathrm{NF}-\kappa \mathrm{B}$ (39). Lipid peroxidation products activate the NF- $\kappa \mathrm{B}$ pathway and inactivate anti-apoptotic B-Cell Lymphoma-2 (Bcl-2) (40,41). The main cellular function of $\mathrm{Bcl}-2$ is the regulation of cytochrome c release $(42,43)$. Thus, the apoptotic cell death induced by OxDHA may be associated with NF- $\kappa$ B activation and the change in the level of Bcl-2.

In summary, we have demonstrated that oxidized fatty acids produced from unsaturated fatty acids play important roles in the apoptotic cell death pathway. The oxidized fatty acid products regulate not only apoptosis, but also non-apoptotic cell death induction (ferroptosis, autophagy, and regulated neutrophil cell death in immune response) (44-47). OxDHA may be useful for studying the mechanism of cell death induced by lipid peroxidation, which is involved in a wide variety of diseases.

\section{Acknowledgements}

Not applicable.

\section{Funding}

Financial support for this research was provided by Grants-in-Aid for Scientific Research of Seikei University (Tokyo, Japan; grant no. 2017) and Scientific Research from the Japan Society for the Promotion of Science (grant nos. $15 \mathrm{~K} 16524$ and $18 \mathrm{~K} 11001$ to KI).

\section{Availability of data and materials}

All data generated and/or analyzed during the current study are available from the corresponding author on reasonable request.

\section{Authors' contributions}

$\mathrm{KI}, \mathrm{ME}, \mathrm{MS}$ and $\mathrm{HH}$ conceived and designed the experiments. $\mathrm{KI}, \mathrm{ME}, \mathrm{MS}$ and CY performed the experiments. KI, ME, MS and $\mathrm{HH}$ contributed to data analysis. KI, ME, MS and $\mathrm{HH}$ contributed to manuscript preparation. All authors read and approved the final manuscript.

\section{Ethics approval and consent to participate}

The present study was approved by the Institutional Review of Committee of Seikei University (Tokyo, Japan). Written informed consent was obtained from volunteers in accordance with the Declaration of Helsinki.

\section{Patient consent for publication}

Not applicable.

\section{Competing interests}

The authors declare that they have no competing interests.

\section{References}

1. Wang W,Zhu J,Lyu F, Panigrahy D, Ferrara KW, Hammock B and Zhang G: $\omega-3$ polyunsaturated fatty acids-derived lipid metabolites on angiogenesis, inflammation and cancer. Prostaglandins Other Lipid Mediat 113-115: 13-20, 2014.

2. Niki E: Biomarkers of lipid peroxidation in clinical material. Biochim Biophys Acta 1840: 809-817, 2014.

3. Calder PC: Polyunsaturated fatty acids and inflammation. Biochem Soc Trans 33: 423-427, 2005.

4. Zajdel A, Wilczok A,Chodurek E, Gruchlik A and DzierzewiczZ: Polyunsaturated fatty acids inhibit melanoma cell growth in vitro. Acta Pol Pharm 70: 365-369, 2013.

5. Yee LD, Young DC, Rosol TJ, Vanbuskirk AM and Clinton SK: Dietary (n-3) polyunsaturated fatty acids inhibit HER-2/neu-induced breast cancer in mice independently of the PPARgamma ligand rosiglitazone. J Nutr 135: 983-988, 2005.

6. Notarnicola M, Tutino V, De Nunzio V, Dituri F, Caruso MG and Giannelli G: Dietary $\omega-3$ polyunsaturated fatty acids inhibit tumor growth in transgenic ApcMin/+ mice, correlating with CB1 receptor Up-Regul. Int J Mol Sci 18: pii: E485, 2017. 
7. Notarnicola M, Messa C, Refolo MG, Tutino V, Miccolis A and Caruso MG: Polyunsaturated fatty acids reduce fatty acid synthase and hydroxy-methyl-glutaryl CoA-reductase gene expression and promote apoptosis in HepG2 cell line. Lipids Health Dis 10: 10, 2011.

8. Yin Y, Sui C, Meng F, Ma P and Jiang Y: The omega-3 polyunsaturated fatty acid docosahexaenoic acid inhibits proliferation and progression of non-small cell lung cancer cells through the reactive oxygen species-mediated inactivation of the PI3K/Akt pathway. Lipids Health Dis 16: 87, 2017.

9. D'Eliseo D and Velotti F: Omega-3 fatty acids and cancer cell cytotoxicity: Implications for multi-targeted cancer therapy. J Clin Med 5: pii: E15, 2016.

10. Pizato N, Luzete BC, Kiffer LFMV, Corrêa LH, de Oliveira Santos I, Assumpção JAF, Ito MK and Magalhães KG: Omega-3 docosahexaenoic acid induces pyroptosis cell death in triple-negative breast cancer cells. Sci Rep 8: 1952, 2018.

11. Mansara PP, Deshpande RA, Vaidya MM and Kaul-Ghanekar R: Differential ratios of omega fatty acids (AA/EPA+DHA) modulate growth, lipid peroxidation and expression of tumor regulatory MARBPs in breast cancer cell lines MCF7 and MDA-MB-231. PLoS One 10: e0136542, 2015.

12. $\mathrm{Mu}$ YM, Yanase T, Nishi Y, Tanaka A, Saito M, Jin $\mathrm{CH}$, Mukasa C, Okabe T, Nomura M, Goto K and Nawata H: Saturated FFAs, palmitic acid and stearic acid, induce apoptosis in human granulosa cells. Endocrinology 142: 3590-3597, 2001.

13. Zhang Y, Xue R, Zhang Z, Yang X and Shi H: Palmitic and linoleic acids induce ER stress and apoptosis in hepatoma cells. Lipids Health Dis 11: 1, 2012.

14. Murray M, Dyari HR, Allison SE and Rawling T: Lipid analogues as potential drugs for the regulation of mitochondrial cell death. Br J Pharmacol 171: 2051-2066, 2014

15. Bochkov VN, Oskolkova OV, Birukov KG, Levonen AL, Binder CJ and Stöckl J: Generation and biological activities of oxidized phospholipids. Antioxid Redox Signal 12: 1009-1059, 2010.

16. Miller YI and Shyy JY: Context-dependent role of oxidized lipids and lipoproteins in inflammation. Trends Endocrinol Metab 28 : 143-152, 2017

17. Clay CE, Monjazeb A, Thorburn J, Chilton FH and High KP: 15-Deoxy-delta12,14-prostaglandin J2-induced apoptosis does not require PPARgamma in breast cancer cells. J Lipid Res 43 : 1818-1828, 2002

18. Chen R, Yang L and McIntyre TM: Cytotoxic phospholipid oxidation products. Cell death from mitochondrial damage and the intrinsic caspase cascade. J Biol Chem 282: 24842-24850, 2007.

19. Wang L, Gill R, Pedersen TL, Higgins LJ, Newman JW and Rutledge JC: Triglyceride-rich lipoprotein lipolysis releases neutral and oxidized FFAs that induce endothelial cell inflammation. J Lipid Res 50: 204-213, 2009.

20. Liu X, Shibata T, Hisaka S, Kawai Y and Osawa T: DHA hydroperoxides as a potential inducer of neuronal cell death: A mitochondrial dysfunction-mediated pathway. J Clin Biochem Nutr 43: 26-33, 2008

21. Lim SY, Jang JH, Na HK, Lu SC, Rahman I and Surh YJ: 15-Deoxy-Delta12,14-prostaglandin $\mathrm{J}(2)$ protects against nitrosative PC12 cell death through up-regulation of intracellular glutathione synthesis. J Biol Chem 279: 46263-46270, 2004.

22. Gao B, Han YH, Wang L, Lin YJ, Sun Z, Lu WG, Hu YQ, Li JQ, Lin XS, Liu BH, et al: Eicosapentaenoic acid attenuates dexamethasome-induced apoptosis by inducing adaptive autophagy via GPR120 in murine bone marrow-derived mesenchymal stem cells. Cell Death Dis 7: e2235, 2016.

23. Yeh A, Kruse SE, Marcinek DJ and Gallagher EP: Effect of omega-3 fatty acid oxidation products on the cellular and mitochondrial toxicity of BDE 47. Toxicol In Vitro 29: 672-680, 2015.

24. Haeiwa H, Fujita T, Saitoh Y and Miwa N: Oleic acid promotes adaptability against oxidative stress in 3T3-L1 cells through lipohormesis. Mol Cell Biochem 386: 73-83, 2014.

25. Iuchi K, Imoto A, Kamimura N, Nishimaki K, Ichimiya H, Yokota $T$ and Ohta S: Molecular hydrogen regulates gene expression by modifying the free radical chain reaction-dependent generation of oxidized phospholipid mediators. Sci Rep 6: 18971, 2016.

26. Abdelmagid SA, Clarke SE, Nielsen DE, Badawi A,El-Sohemy A Mutch DM and Ma DW: Comprehensive profiling of plasma fatty acid concentrations in young healthy Canadian adults. PLoS One 10: e0116195, 2015

27. Eguchi Y, Shimizu S and Tsujimoto Y: Intracellular ATP levels determine cell death fate by apoptosis or necrosis. Cancer Res 57: 1835-1840, 1997
28. Schley PD, Jijon HB, Robinson LE and Field CJ: Mechanisms of omega-3 fatty acid-induced growth inhibition in MDA-MB-231 human breast cancer cells. Breast Cancer Res Treat 92: 187-195, 2005.

29. Perluigi M, Coccia R and Butterfield DA: 4-Hydroxy-2-nonenal, a reactive product of lipid peroxidation, and neurodegenerative diseases: A toxic combination illuminated by redox proteomics studies. Antioxid Redox Signal 17: 1590-1609, 2012.

30. Milne GL, Yin H, Hardy KD, Davies SS and Roberts LJ II: Isoprostane generation and function. Chem Rev 111: 5973-5996, 2011.

31. Usatyuk PV and Natarajan V: Hydroxyalkenals and oxidized phospholipids modulation of endothelial cytoskeleton, focal adhesion and adherens junction proteins in regulating endothelial barrier function. Microvasc Res 83: 45-55, 2012.

32. Esterbauer H, Schaur RJ and Zollner H: Chemistry and biochemistry of 4-hydroxynonenal, malonaldehyde and related aldehydes. Free Radic Biol Med 11: 81-128, 1991.

33. Meilhac O, Zhou M, Santanam N and Parthasarathy S: Lipid peroxides induce expression of catalase in cultured vascular cells. J Lipid Res 41: 1205-1213, 2000.

34. Nourooz-Zadeh J, Liu EH, Anggård E and Halliwell B: F4-isoprostanes: A novel class of prostanoids formed during peroxidation of docosahexaenoic acid (DHA). Biochem Biophys Res Commun 242: 338-344, 1998.

35. Majkova Z, Layne J, Sunkara M, Morris AJ, Toborek M and Hennig B: Omega-3 fatty acid oxidation products prevent vascular endothelial cell activation by coplanar polychlorinated biphenyls. Toxicol Appl Pharmacol 251: 41-49, 2011.

36. Martínez B, Pérez-Castillo A and Santos A: The mitochondrial respiratory complex I is a target for 15-deoxy-delta12,14-prostaglandin J2 action. J Lipid Res 46: 736-743, 2005.

37. Kang KS, Wang P, Yamabe N, Fukui M, Jay T and Zhu BT: Docosahexaenoic acid induces apoptosis in MCF-7 cells in vitro and in vivo via reactive oxygen species formation and caspase 8 activation. PLoS One 5: e10296, 2010.

38. Yun EJ, Song KS, Shin S, Kim S, Heo JY, Kweon GR, Wu T, Park JI and Lim K: Docosahexaenoic acid suppresses breast cancer cell metastasis by targeting matrix-metalloproteinases. Oncotarget 7: 49961-49971, 2016.

39. Dwarakanath RS, Sahar S, Reddy MA, Castanotto D, Rossi JJ and Natarajan R: Regulation of monocyte chemoattractant protein-1 by the oxidized lipid, 13-hydroperoxyoctadecadienoic acid, in vascular smooth muscle cells via nuclear factor-kappa B (NF-kappa B). J Mol Cell Cardiol 36: 585-595, 2004.

40. Timucin AC and Basaga H: Pro-apoptotic effects of lipid oxidation products: $\mathrm{HNE}$ at the crossroads of NF- $\kappa \mathrm{B}$ pathway and anti-apoptotic Bcl-2. Free Radic Biol Med 111: 209-218, 2017.

41. Yadav UC and Ramana KV: Regulation of NF- $\kappa B$-induced inflammatory signaling by lipid peroxidation-derived aldehydes. Oxid Med Cell Longev 2013: 690545, 2013.

42. Tsujimoto Y: Cell death regulation by the Bcl-2 protein family in the mitochondria. J Cell Physiol 195: 158-167, 2003

43. Shimizu S and Tsujimoto Y: Proapoptotic BH3-only Bcl-2 family members induce cytochrome c release, but not mitochondrial membrane potential loss, and do not directly modulate voltage-dependent anion channel activity. Proc Natl Acad Sci USA 97: 577-582, 2000

44. Hill BG, Haberzettl P, Ahmed Y, Srivastava S and Bhatnagar A: Unsaturated lipid peroxidation-derived aldehydes activate autophagy in vascular smooth-muscle cells. Biochem J 410: 525-534, 2008

45. Xie Y, Hou W, Song X, Yu Y, Huang J, Sun X, Kang R and Tang D: Ferroptosis: Process and function. Cell Death Differ 23: 369-379, 2016.

46. Yotsumoto S, Muroi Y, Chiba T, Ohmura R, Yoneyama M, Magarisawa M, Dodo K, Terayama N, Sodeoka M, Aoyagi R, et al: Hyperoxidation of ether-linked phospholipids accelerates neutrophil extracellular trap formation. Sci Rep 7: 16026, 2017.

47. Parisi LR, Morrow LM, Visser MB and Atilla-Gokcumen GE: Turning the spotlight on lipids in non-apoptotic cell death. ACS Chem Biol 13: 506-515, 2018.

This work is licensed under a Creative Commons Attribution-NonCommercial-NoDerivatives 4.0 International (CC BY-NC-ND 4.0) License. 